\title{
IMPORTANCIA DE LA IMPLEMENTACIÓN DE UN MODELO DE GESTIÓN EN LA PESCA ARTESANAL DEL CANTÓN MANTA, ECUADOR
}

\author{
IMPORTANCE OF THE IMPLEMENTATION OF A MANAGEMENT MODEL IN THE TRADITIONAL \\ FISHING CANTON MANTA, ECUADOR.
}

\author{
Juan Carlos Lara Ocaña* \\ jclaraocana1961@outlook.com \\ Mario Moreira Moreira*** \\ mmoreira.moreira3@gmail.com \\ Guadalupe Toala Toala**** \\ faccauleam@gmail.com \\ Evelyn Cano Lara******* \\ evelyn.cano@uleam.edu.ec
}

[RECEPCIÓN: AGOSTO 2015 / CONFORMIDAD: NOVIEMBRE 2015]

\section{RESUMEN}

Un importante grupo de la población ecuatoriana está ligado a la pesca artesanal donde se origina la mayoría del pescado fresco que se consume en el país. En el cantón Manta existen registradas aproximadamente 5,000 embarcaciones conocidas como fibras, las cuales son esenciales para la pesca; sin embargo, en los últimos cuatro años, los pescadores artesanales han tenido que soportar ingentes pérdidas económicas por el robo de sus aparejos de pesca y motores en alta mar, situación que cobra a veces hasta la vida de los pescadores. La investigación se basa en el análisis situacional de la pesca artesanal y la problemática existente en la gestión de sus actividades, proponiendo la implementación de un modelo de gestión que disminuya la piratería en alta mar y solucione otros inconvenientes relacionados con esta. De modo que el objetivo de este trabajo es proponer un modelo que mejore la gestión de la pesca artesanal en el Cantón Manta.

Palabras clave: Gestión, pesca artesanal, modelos de gestión, sistema de monitoreo y control.

* Estudiante del Doctorado en Ciencias Administrativas de la Universidad Nacional Mayor de San Marcos (UNMSM). Ex director financiero, actualmente docente de la Facultad de Economía de la Universidad Laica Eloy Alfaro de Manabí (Ecuador). Presidente de la Asociación de Funcionarios del Área Financiera de las Universidades y Escuelas Politécnicas del Ecuador.

** Estudiante del Doctorado en Ciencias Administrativas (UNMSM). Ex Decano de la Facultad de Ciencias Administrativas de la Universidad Laica Eloy Alfaro de Manabí (Ecuador), actualmente docente en dicha facultad.

****** Estudiante del Doctorado en Ciencias Administrativas (UNMSM). Ex directora de Escuela de la Facultad de Ciencias Administrativas de la Universidad Laica Eloy Alfaro de Manabí (Ecuador), actualmente docente en dicha facultad.

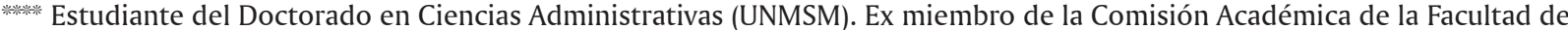
Ciencias Administrativas de la Universidad Laica Eloy Alfaro de Manabí (Ecuador), actualmente docente en dicha facultad. 


\begin{abstract}
An important group of the Ecuadorian population is linked to the artisanal fisheries where most of the fresh fish consumed in the country originates. In the canton Manta, there are approximately 5,000 registered boats known as fibers, which are essential for fishing; however, in the last four years artisanal fishermen have endured massive economic losses by theft of fishing gear and engines at sea, a situation that sometimes it's even mortal for the crew. The research is based on the situational analysis of artisanal fisheries and the existing problems in the management of its activities, proposing the implementation of a management model to decrease piracy on the high seas and solve other problems related to it. The main Objective of this work is to propose a model to improve the management of artisanal fisheries in Canton Manta.
\end{abstract}

Keywords: Management, artisanal fisheries management models, monitoring and control.

\section{INTRODUCCIÓN}

La ciudad de Manta ha tenido un crecimiento económico acelerado en los últimos años, esto se debe a que su puerto marítimo posee una gran ventaja, en relación con los otros puertos del país por el ingreso directo de buques de gran calado a sus muelles, por la gran actividad industrial que está sustentada directa y preferentemente con la pesca y el efecto positivo que ocasiona la comercialización de sus productos elaborados, los cuales tienen una fuerte demanda en el mercado externo.

Un importante grupo de la población ecuatoriana está ligado a la pesca artesanal donde se origina la mayoría del pescado fresco que se consume en el país (Escuela Superior Politécnica del litoral, ESPOL, 1987); existen 57 puertos pesqueros artesanales, alrededor de 11,000 pescadores asentados en la costa y cerca de 2,200 personas intervienen en la comercialización nacional de productos del mar. En el cantón Manta existen registradas aproximadamente 5,000 embarcaciones conocidas como fibras, las cuales son esenciales para la realización de esta actividad, ya que mínimo tres pescadores salen a sus faenas en una fibra (Reyes, 2012); sin embargo, en los últimos cuatro años los pescadores artesanales han tenido que soportar ingentes pérdidas económicas por el robo de sus aparejos de pesca y motores en alta mar, situación que cobra a veces hasta la vida. Este hecho guarda relación con la culminación del convenio entre los Estados Unidos y Ecuador para que funcione en Manta el Puesto de Operaciones Avanzadas ${ }^{1}$, quienes controlaban las actividades en las embarcaciones dedicadas a la pesca artesanal, una vez retirada se dio espacio para la ejecución de actividades ilegales.(El Diario Manabita del libre pensamiento, 2008).
Esta problemática hace un llamado a corregir las prácticas tradicionales en la pesca artesanal, la misma que está directamente relacionada con los nuevos objetivos, fundamentos, diagnóstico, políticas y lineamientos, línea base y metas dictadas por el Estado Central, en la búsqueda del buen vivir; no obstante la seguridad ciudadana no ha sido considerada para el sector de la pesca artesanal; de hecho, este sector ha estado completamente abandonado incluso en el ordenamiento de sus actividades laborales y sociales. El Estado Central a través de sus instituciones como Capitanía del Puerto, Ministerio de Justicia, tienen la potestad legal de buscar soluciones y el Gobierno local debe precautelar además de la seguridad de sus ciudadanos, la de los visitantes que realizan actividades turísticas, sean éstos nacionales o extranjeros.

\section{JUSTIFICACIÓN}

Se propone la creación de un modelo de gestión para mejorar la pesca artesanal del cantón Manta, ya que éste sería conveniente por las siguientes razones:

- Un modelo de gestión afectará directamente en el empoderamiento de las responsabilidades que tienen los actores, en mejorar los procesos de zarpe, rutas y pesca en alta mar y traslado y venta de la misma.

- Aportará al cambio de los conceptos teóricos sobre el control y seguridad de las gestiones relacionadas con la pesca, a través de la implementación de medios tecnológicos y de la creación de normas que regulen su uso.

- Se establecería la creación de un Centro Público de Monitoreo y Seguridad para realizar

1 Base militar conocida como FOL por sus siglas en inglés. 
el seguimiento de las actividades de pesca de cada embarcación.

A su vez sería de utilidad para la Armada del Ecuador, la Fiscalía General del Estado, los armadores pesqueros ${ }^{2}$, los tripulantes y las familias de los tripulantes quienes al ser informados de las faenas realizadas en alta mar estarían seguros que sus esposos, padres, hijos o nietos, se encuentran laborando con normalidad.

Los sistemas de monitoreo digitalizados son herramientas muy necesarias en la sociedad para solucionar problemas de inseguridad; actualmente la mayoría de las ciudades en el mundo poseen estos sistemas de monitoreo digitalizados para controlar el comportamiento del ciudadano, el tránsito urbano y el interior de los vehículos de pasajeros.

\section{MARCO FILOSÓFICO DE LA INVESTIGACIÓN}

Durante la historia se han presentado diferentes propuestas de modelos de gestión entre las que puedo mencionar resumidamente:

El modelo japonés - modelo Deming (1951)

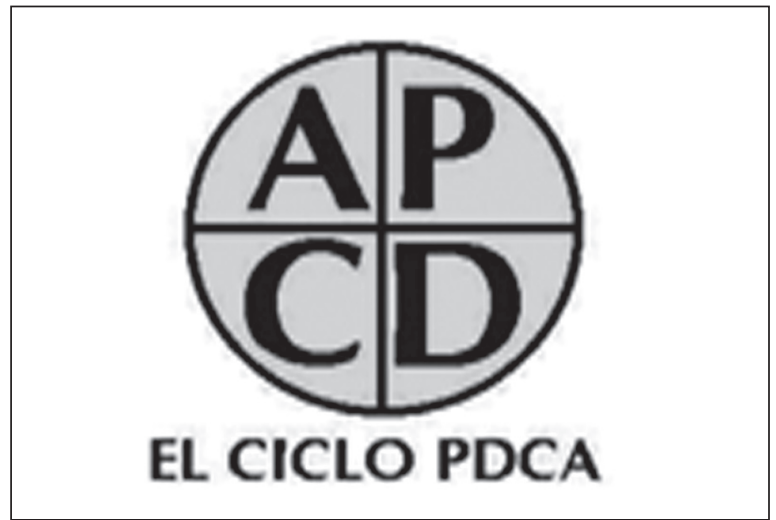

Figura $\mathrm{N}^{\circ} 1$. Resume los ideales del modelo Deming

El Modelo de Deming estudia cómo realizar las políticas de dirección, control y de calidad, y cómo se transmiten éstas a todos los sectores de la empresa. Este modelo recoge la aplicación práctica de las teorías japonesas del control total de la calidad (TQC) o control de calidad en toda la empresa (CWQC).

Desarrollado en Japón en 1951 por la Unión Japonesa de Científicos e Ingenieros (JUSE) en 1951, presenta una metodología de gerencia de la calidad, basada principalmente en la generación de una planificación empresarial a partir del liderazgo del ejecutivo o dueño de la empresa, que permite realizar emprendimientos en varias áreas, una vez que se haya preparado idóneamente al personal.

Según Deming, la buena organización de la empresa dependerá de la evaluación realizada a cada uno de los departamentos que permitan visualizar claramente que la ejecución de lo planificado se realice con responsabilidad y acato a las disposiciones de la autoridad entre los jefes de los diferentes departamentos de la empresa para promover entre ellos la cooperación. Este modelo basa sus ideales en planificar, hacer, verificar y actuar.

\section{El Modelo Europeo de Excelencia EFQM (1992)}

Se crea un nuevo modelo de gestión en Europa en el año 1992, por efectos de la imperiosa necesidad de mejorar la gestión en la educación y estar a nivel de la competencia proveniente de Estados Unidos y Japón. Básicamente este modelo actúa bajo el mando de en equipo gestor capacitado (lideres) y tiene un impacto sobre los clientes y la sociedad en general.

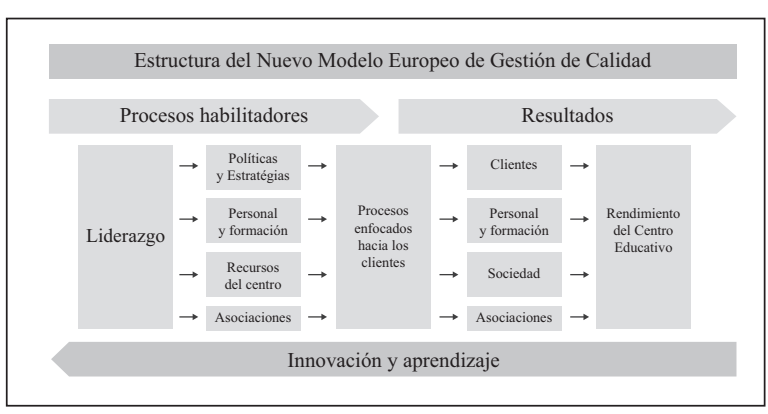

Figura №2. Estructura del Nuevo Modelo Europeo de Gestión de Calidad

Los criterios básicos del modelo son nueve: cinco de los criterios se relacionan con el liderazgo, planificación y estrategia, además de los colaboradores, recursos y procesos; los cuatro restantes se refieren a los resultados en el entorno de la organización.

Este modelo identifica que el eje principal de toda empresa es la capacidad de liderazgo que tienen los integrantes del equipo directivo, ya que ellos desarrollan las políticas necesarias para alcanzar el éxito programado y realizan planes estratégicos basados en objetivos que los llevarán a alcanzar la excelencia.

El Modelo Europeo de Gestión y Calidad busca que la organización se enfoque en los resultados

2 Dueños de las embarcaciones artesanales. 
de satisfacción de los clientes, de los resultados de la evaluación del personal y la afectación de las actividades de la organización en la sociedad, en la búsqueda de un excelente rendimiento final.

\section{Modelo de Excelencia en la Gestión de Mal- colm Baldrige (1987)}

Baldrige propone en su Modelo de Excelencia en la Gestión la mejora de las prácticas de gestión a través de la identificación plena del liderazgo que debe tener el que dirige la organización. Busca además la capacitación del recurso humano como una contante en la organización y evalúa los resultados de los procesos; por esta razón, se identifica a este modelo como el de las autoevaluaciones.

El Modelo de Excelencia resalta el valor de los clientes y reconoce a estos como el sostén de la organización, instruye a través de las capacitaciones el mejoramiento permanente de la eficacia y las capacidades de cada uno de sus integrantes.

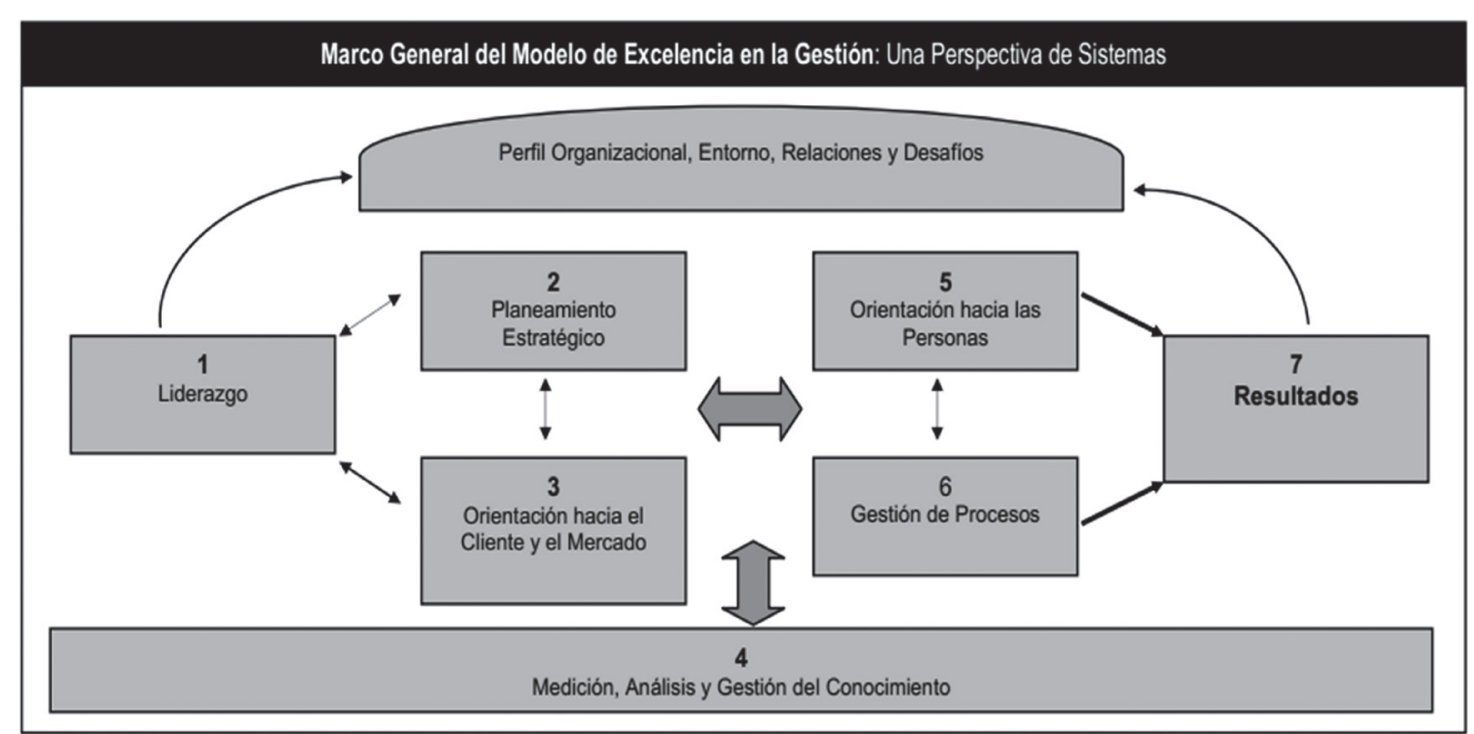

Figura $N^{\circ}$ 3. Marco General del Modelo de Excelencia en la Gestión de Baldrige

El Modelo de Excelencia de Baldrige trata de empoderar en el líder el conocimiento del perfil organizacional, el entorno de la organización y el mantenimiento de las relaciones adecuadas que le permitan conocer los desafíos futuros de manera oportuna.

Gestión Sostenible de la Pesca, Freire (2002)

Freire (2002) en Gestión sostenible de la pesca, indica la existencia del modelo de Gestión Clásica y Modelo de Gestión de Ecosistemas, en el cual el modelo de Gestión Clásica prioriza el rendimiento máximo sostenible utilizando el criterio del MSY (maximum sustainable yield); en cambio el modelo de Gestión de Ecosistemas propone la consideración no solamente de la variable bienes, sino introduce el concepto de servicios, lo cual tiene mucha razón pues la pesca artesanal no es solamente el intercambio de productos del mar por dinero ya que en la actualidad se deben gestionar procesos que permitan la conservación de la pesca, considerando a esta actividad como un servicio independiente.

\begin{tabular}{|c|c|}
\hline GESTIÓN "CLÁSICA" & GESTIÓN DE ECOSISTEMAS \\
\hline $\begin{array}{l}\text { - Valores utilitarios } \\
\text { - Producción de bienes (MSY) } \\
\text { - Gestión y modelos de especies } \\
\text { individuales } \\
\text { - Modelos científicos deterministas } \\
\text { - Gobierno "top-down" basado en expertos } \\
\text { y administración pública } \\
\text { - Monopolio científico de datos y análisis } \\
\text { - "Social" = nivel de uso del recurso }\end{array}$ & $\begin{array}{l}\text { - Valores utilitarios y éticos } \\
\text { - Bienes y servicios } \\
\text { - Múltiples especies, hábitat, interacciones } \\
\text { - Gestión bioregional y adaptiva } \\
\text { - Ciencia acepta la incertidumbre } \\
\text { - Gobierno "bottom-up" colaborativo } \\
\text { - "Social" = activo, grupos de usuarios } \\
\text { y comunidades involucrados activamente }\end{array}$ \\
\hline
\end{tabular}

Figura $N^{\circ} 4$. Gestión sostenible de la pesca. Análisis comparativo entre la gestión clásica y la gestión de ecosistemas

Actualmente los pescadores artesanales no se dedican a la pesca de una sola variedad de pescado; de acuerdo a la temporada cambian los aparejos de pesca a efectos de estar activos durante todo el año en el desarrollo de sus acciones pesqueras. Además se debe considerar que las autoridades gubernamentales decretan periodos de veda para 
ciertas especies, especialmente los pelágicos lo que obliga al cambio de la variedad de pescado.

\section{METODOLOGÍA}

\section{Tipo y diseño de investigación}

Esta investigación es de tipo cualitativo porque se hacen registros narrativos de los fenómenos que son estudiados mediante técnicas como la encuesta; es correlacional pues persigue medir el grado de relación o vínculo (no causal) existente entre dos o más variables.

\section{Unidad de análisis}

La unidad de análisis serán los pescadores y los armadores artesanales.

\section{Instrumento de recolección de datos}

Para la obtención de datos se utilizó la técnica de la encuesta, la misma que se aplicó a una muestra de 1,560 pescadores y 60 armadores, distribuidos de la siguiente manera:

\section{Cuadro $\mathrm{N}^{\circ} 1$. Distribución de la muestra}

\begin{tabular}{|c|c|c|c|c|}
\hline CONCEPTO & $\begin{array}{c}\text { MANTA } \\
(70 \%)\end{array}$ & $\begin{array}{c}\text { SAN MATEO } \\
(20 \%)\end{array}$ & $\begin{array}{c}\text { SAN } \\
\text { LORENZO } \\
(10 \%)\end{array}$ & TOTAL \\
\hline Pescadores & 1,092 & 312 & 156 & 1,560 \\
\hline Armadores & 44 & 13 & 6 & 63 \\
\hline
\end{tabular}

\section{Procedimiento de recolección de datos}

Las encuestas se aplicaron personalmente a los pescadores y armadores en sus lugares de trabajo, dando la oportunidad de conocer más sobre la actividad y las falencias en las que se desarrolla la pesca artesanal.

\section{PRESENTACIÓN DE RESULTADOS}

\section{Encuesta a los pescadores}

Una vez aplicada las encuestas se obtuvieron los siguientes datos:

Cuadro N. ${ }^{\circ}$. Existencia de centro público

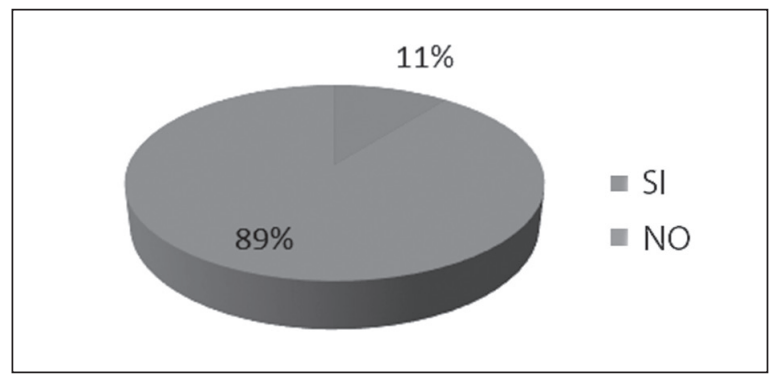

Fuente: Elaboración propia
Cuadro N. $^{\circ} 2$. ¿Labora en una fibra?

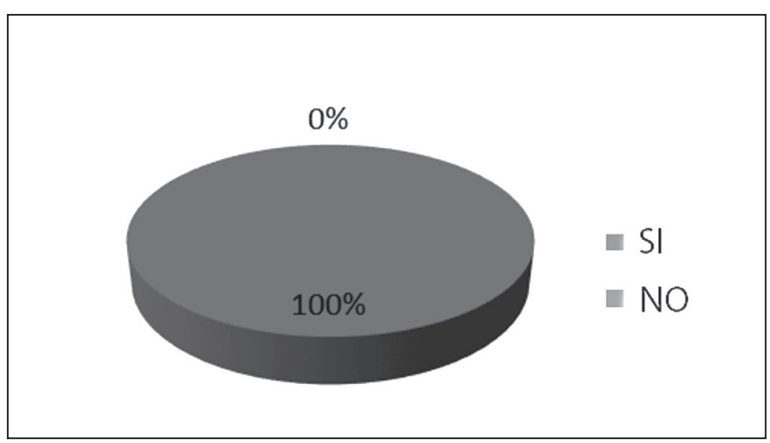

Fuente: Elaboración propia

Cuadro N. ${ }^{\circ} 3$. ¿Cuenta con sistema de monitoreo su fibra?

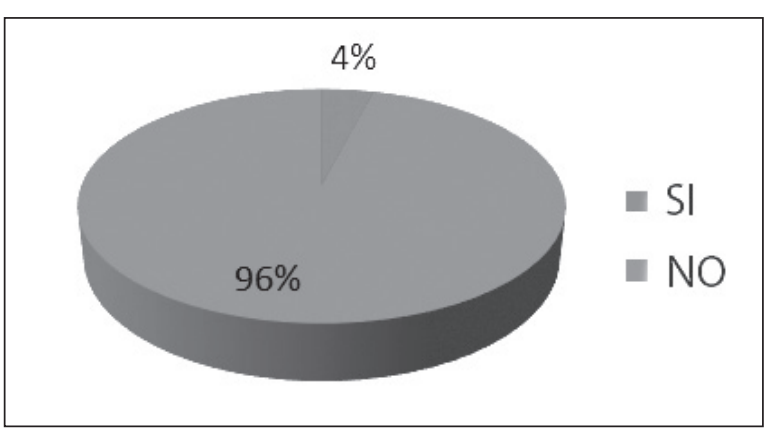

Fuente: Elaboración propia

Cuadro N. ${ }^{\circ} 4$. ¿Le han robado su fibra en alta mar?

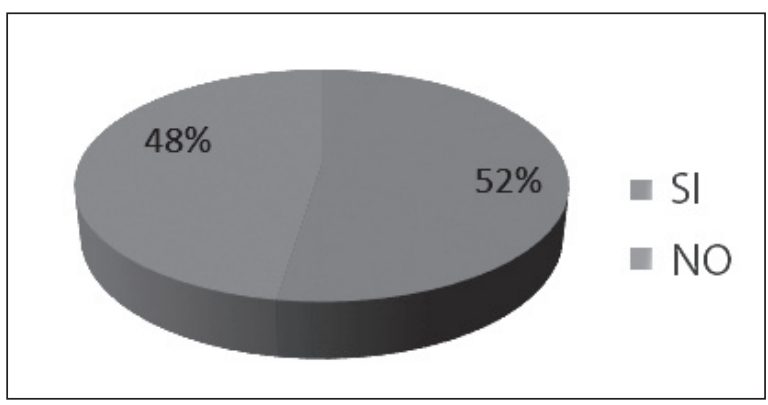

Fuente: Elaboración propia

Cuadro N. ${ }^{\circ}$ 5. ¿Ha sufrido el robo de sus aparejos de pesca?

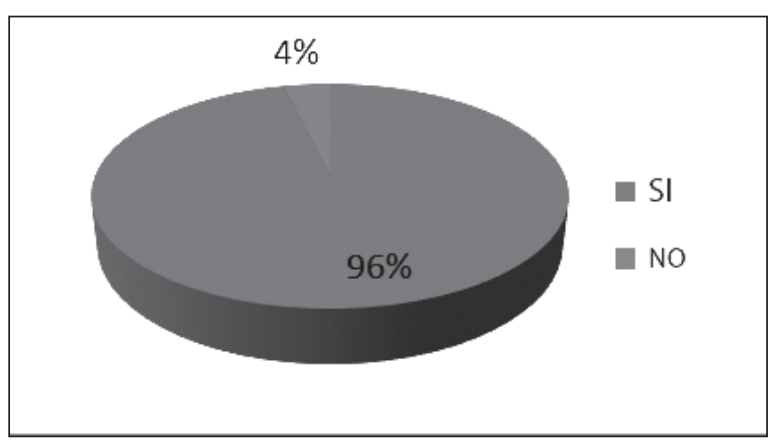

Fuente: Elaboración propia 
Cuadro N. ${ }^{\circ} 6$. ¿Ha sufrido el robo del motor?

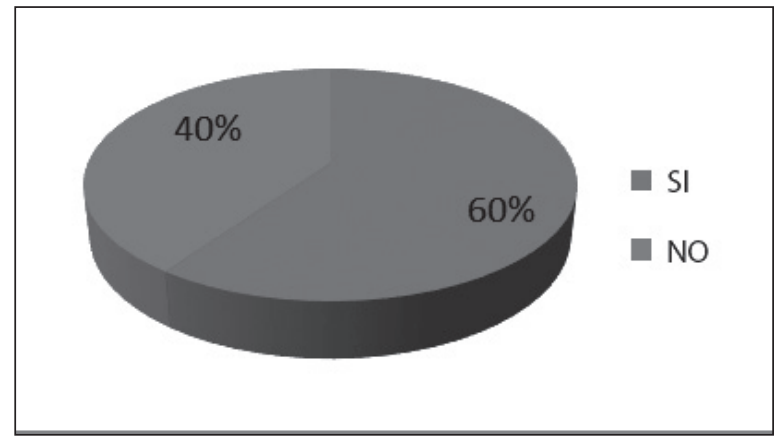

Fuente: Elaboración propia

Cuadro N. ${ }^{\circ} 7$. ¿Ha sufrido el robo de combustible?

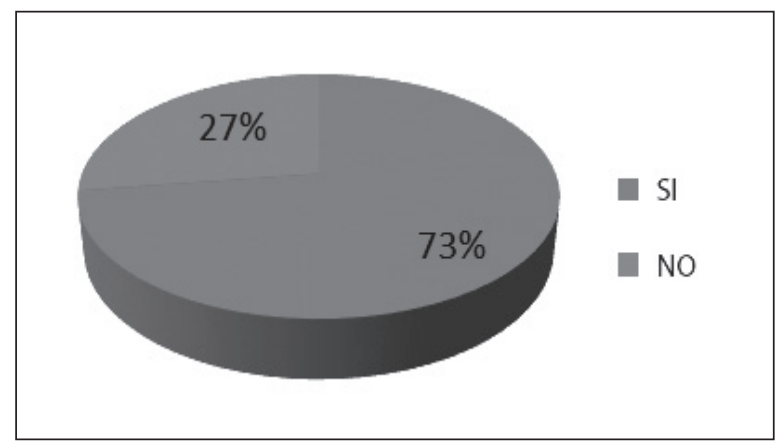

Fuente: Elaboración propia

Cuadro N. ${ }^{\circ} 8$. ¿Conoce usted de la muerte de pescadores a causa de la piratería?

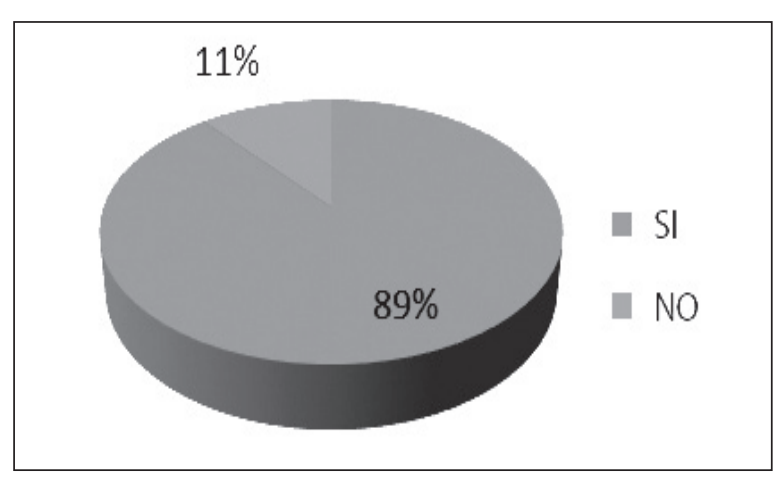

Fuente: Elaboración propia

\section{Encuesta a los armadores}

Cuadro N. ${ }^{\circ}$. Existencia de centro público

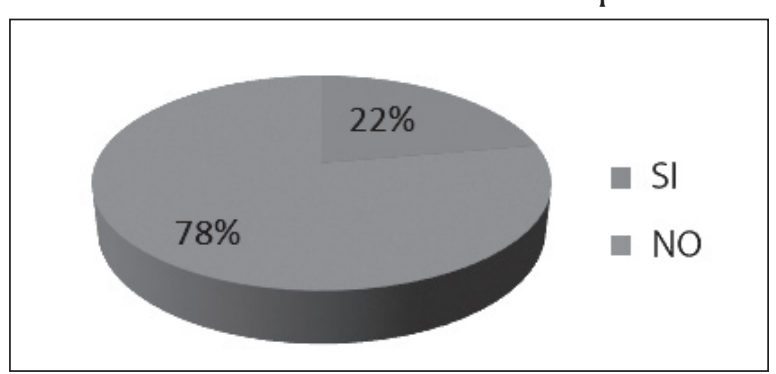

Fuente: Elaboración propia
Cuadro N. ${ }^{\circ} 10$. ¿Mejorará la gestión de la pesca artesanal con la implementación de un centro de monitoreo y seguridad?

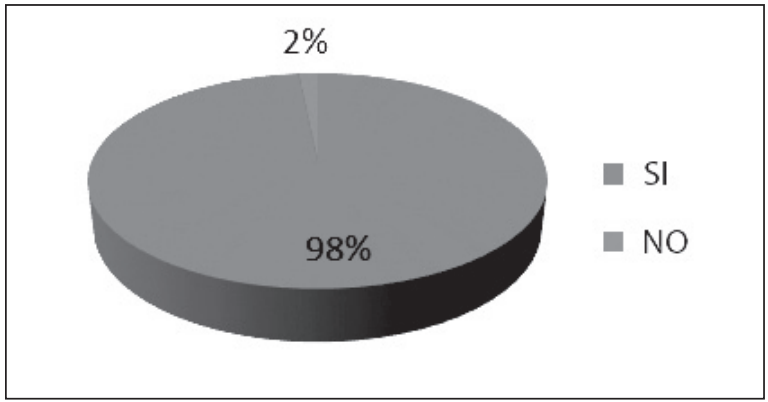

Fuente: Elaboración propia

Cuadro N. ${ }^{\circ} 11$. ¿Tiene su fibra un sistema de monitoreo y seguridad?

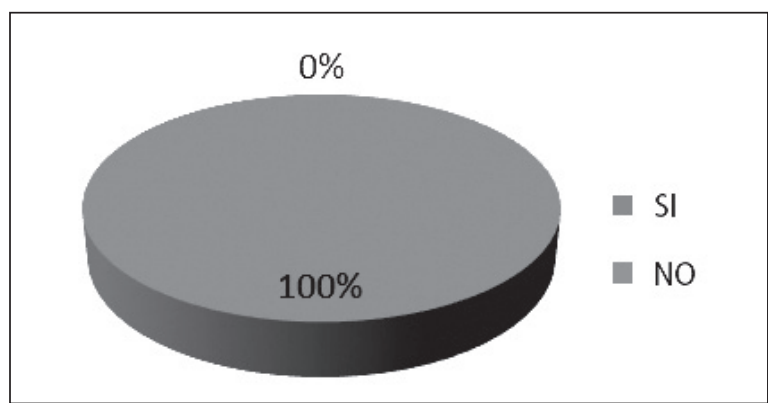

Fuente: Elaboración propia

Cuadro N. ${ }^{\circ} 12$. ¿Le han robado su fibra en alta mar?

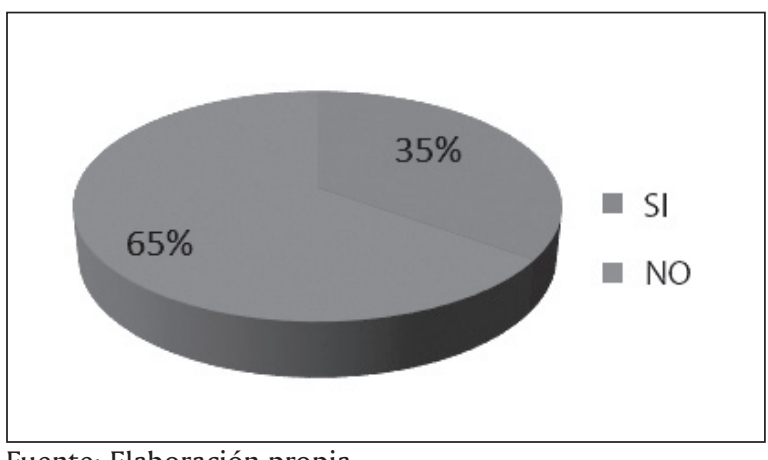

Fuente: Elaboración propia

Cuadro N. ${ }^{\circ} 13$. ¿Ha sufrido la pérdida de los aparejos de pesca?

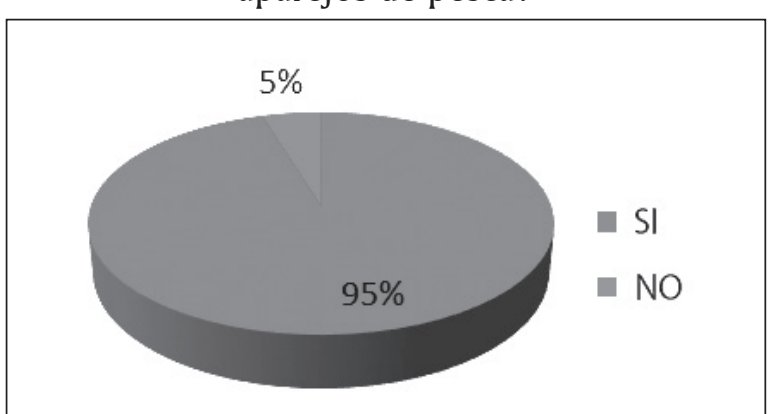

Fuente: Elaboración propia 
Cuadro N. ${ }^{\circ} 14$. ¿Ha sufrido el robo del motor?

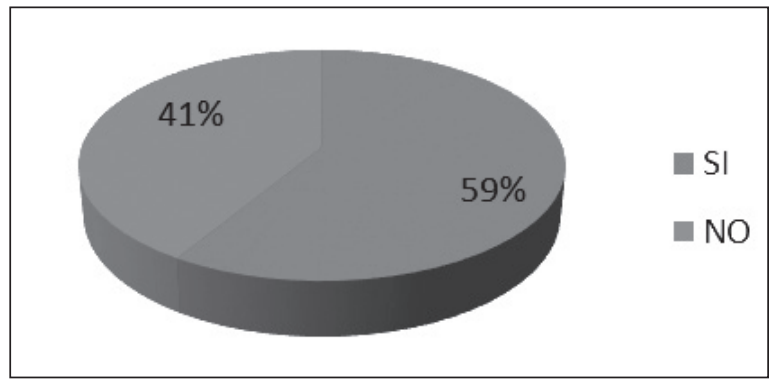

Fuente: Elaboración propia

Cuadro N. ${ }^{\circ} 15$. ¿Ha sufrido el robo del combustible?

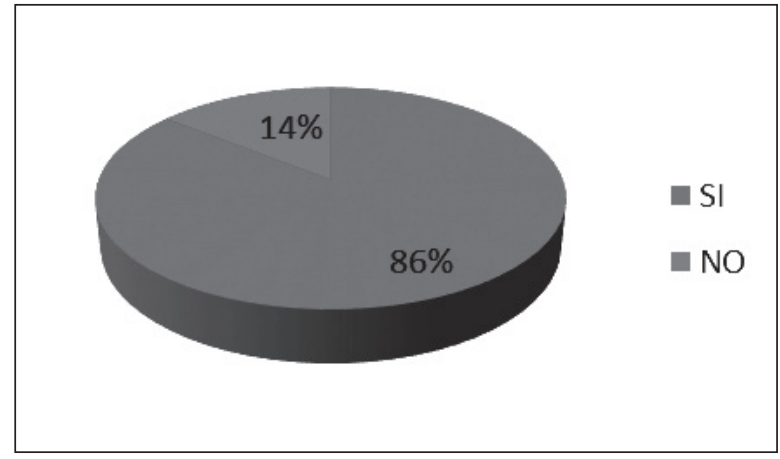

Fuente: Elaboración propia

Cuadro N. ${ }^{\circ} 16$. ¿Conoce usted de la muerte de pescadores a causa de la piratería?

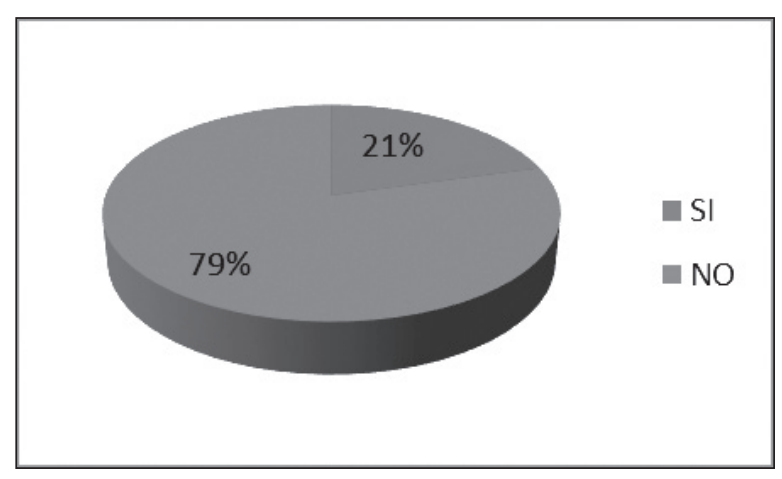

Fuente: Elaboración propia

Cuadro N. ${ }^{\circ} 17$. ¿Tiene un control de horarios de las faenas?

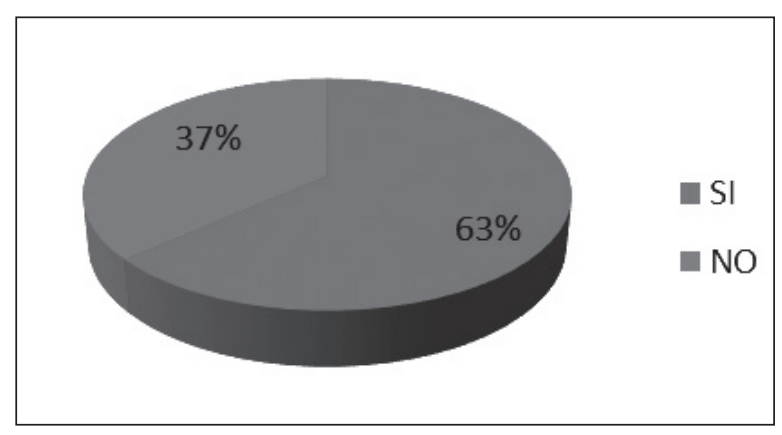

Fuente: Elaboración propia
Cuadro N. ${ }^{\circ} 18$. ¿La instalación de cámaras mejorará el control de la pesca?

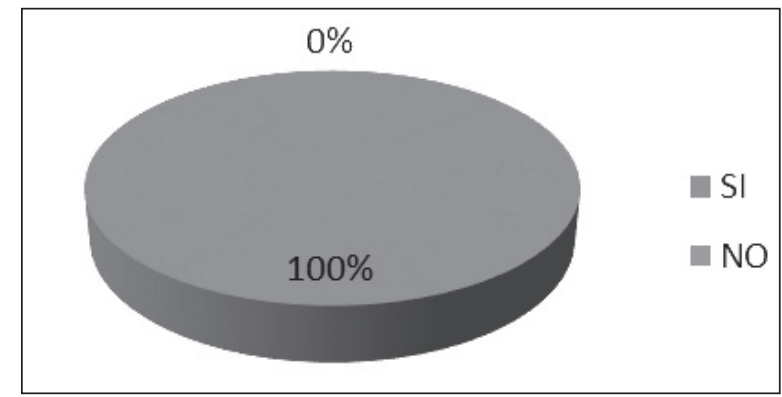

Fuente: Elaboración propia

Cuadro N. ${ }^{\circ} 19$. ¿Un sistema de control evitaría la venta fraudulenta?

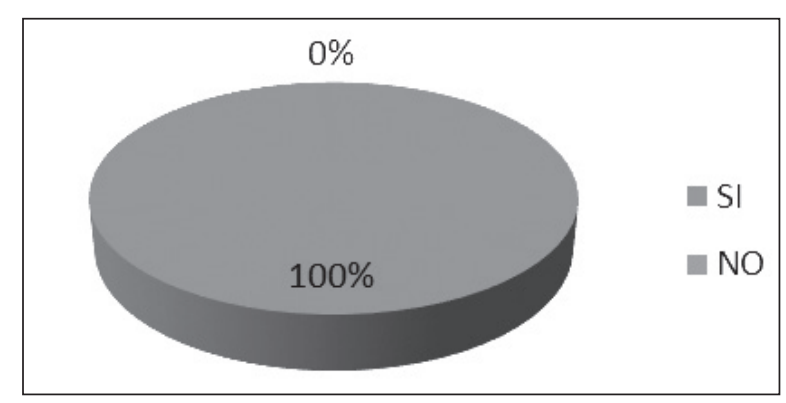

Fuente: Elaboración propia

Cuadro N. ${ }^{\circ} 20$. Al conocer la cantidad de pesca ¿incrementaría sus ingresos?

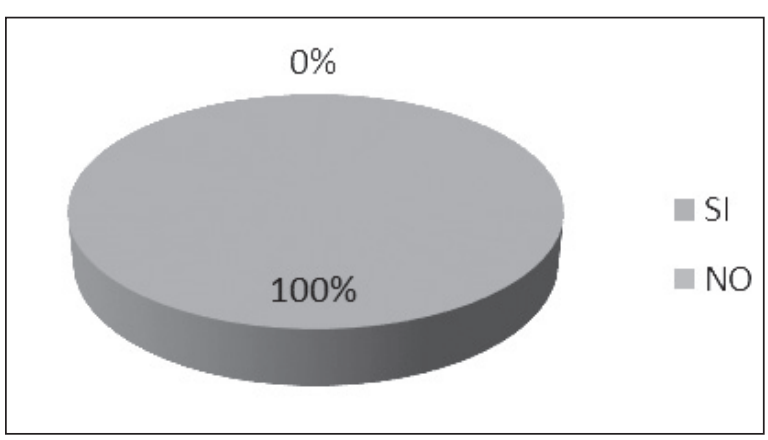

Fuente: Elaboración propia

Cuadro N. ${ }^{\circ} 21$. ¿Un sistema de control permitiría la atención oportuna de emergencias?

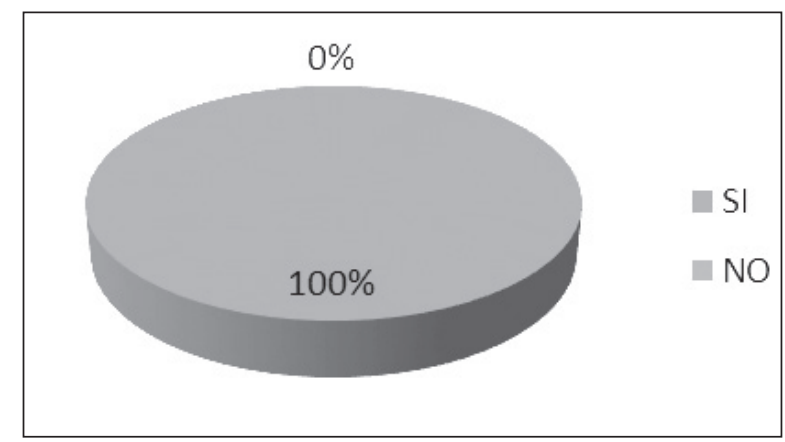

Fuente: Elaboración propia 
Cuadro N. ${ }^{\circ 22}$. ¿Con un sistema de control usted denunciaría a los piratas?

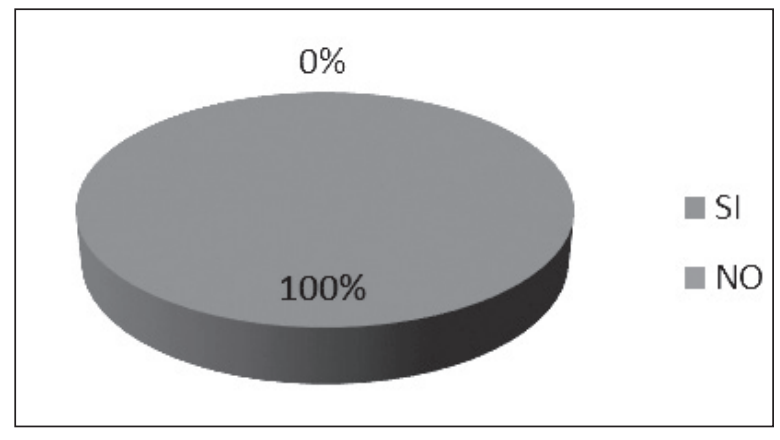

Fuente: Elaboración propia

\section{RESULTADOS Y DISCUSIÓN}

- La representación gráfica de la información recolectada, nos permite interpretar mejor los resultados para conocer la situación actual de la pesca artesanal, cumpliendo con el objetivo específico de la investigación. Al analizar los resultados podemos determinar que:

- Los pescadores y armadores en la actualidad confirman con más de un $70 \%$ la inexistencia de alguna institución que controle y monitoree las faenas de pesca en alta mar; ya que el 100\% de ellos utilizan embarcaciones llamadas fibras para el desarrollo de sus actividades.

- El 96\% de los pescadores no cuentan con un sistema de seguridad, por lo que el $48 \%$ ha sufrido el robo de sus embarcaciones, el $96 \%$ ha perdido los aparejos de pesca, al $60 \%$ le han hurtado su motor, el $73 \%$ lo han despojado de su combustible y un $89 \%$ de los pescadores conoce de la muerte de un familiar u otro pescador por efectos de la piratería en altamar.

- El 100\% de los armadores indican que sus embarcaciones no cuentan con ningún sistema de seguridad, así mismo un 35\% ha sufrido el robo de sus fibras, un $95 \%$ ha perdido los aparejos de pesca, a un $59 \%$ le han hurtado su motor, un $86 \%$ lo han despojado de su combustible y un $21 \%$ de los armadores conoce de la muerte de un familiar u otro pescador por efectos de la piratería.

- Un 98\% de los armadores concuerdan que con la implementación de un sistema de monitoreo y control de la pesca artesanal, se mejoría la gestión de esta actividad; a su vez se controlaría la pesca en alta mar, se dismi- nuiría la venta fraudulenta que favorecería a los ingresos de los pescadores, las emergencias se atenderían con mayor efectividad, se podría denunciar casos de piratería y sobre todo las familias podrían gozar de tranquilidad y seguridad.

\section{CONCLUSIONES}

1. A pesar de ser la pesca artesanal una labor que involucra a un importante grupo de la población ecuatoriana donde se origina la mayoría del pescado fresco que se consume en el país, no ha sido atendida oportunamente ni se ha registrado preocupación por mejorar la situación en la que viven los pescadores y armadores del cantón.

2. Es alto el número de incidencias presentadas en la realización de la pesca artesanal, que van desde robo de la embarcación, los implementos para operar, los aparejos de pesca y hasta la muerte de los trabajadores; siendo racional en estas situaciones no hay una entidad que controle y supervise las actividades en alta mar.

3. Para lograr una mayor eficiencia en la gestión de la pesca artesanal se requiere implementar un sistema de control y seguridad que monitoree las actividades en altamar para salvaguardar la vida de los pescadores y sus faenas; por lo tanto se requiere de un modelo de gestión para la pesca artesanal ejecutado por un centro público que logre cambiar la situación actual en la que viven estos trabajadores.

4. Se prevé la utilización de tecnología y equipos de monitoreo para la vigilancia de las fibras y control en los procesos de pesca en las faenas registradas en una carga horaria que permita la efectividad de la implementación y puesta en marcha del modelo de gestión propuesto con impacto positivo para pescadores y armadores del cantón.

\section{REFERENCIAS BIBLIOGRÁFICAS}

El Diario Manabita del libre pensamiento. 2008. "La pesca artesanal pilar de la economía del cantón”. En: El Diario Manabita del libre pensamiento. 02 de noviembre del 2008. Recuperado de: < http://www.eldiario.ec/noticiasmanabi-ecuador/97211-la-pesca-artesanal-pilarde-la-economia-del-canton/> . 
Escuela Superior Politécnica del Litoral (ESPOL). 1987. La Pesca artesanal en el Ecuador. Recuperado de: <http:/books.google.es/books? id $=$ Xi4RAAAAYAAJ\&q= mercado + laboral $\&$ hl $=$ es \&source $=$ gbs_word_cloud_r\&cad $=4>$.

Freire, Juan (2002). Gestión sostenible de la pesca. Jornadas sobre la Pesca Responsable y el Medio Ambiente Litoral. Universidad de A. Coruña.

Reyes, Carolina (2012). "Manta, primer puerto pesquero del país". En: El Costanero. 20 de enero del 2012. Recuperado de: <http://elcostanero. blogspot.com/2012/01/manta-primer-puertopesquero-del-pais.html $>$. 